\title{
A NOTE ON EXISTENCE AND UNIQUENESS OF SOLUTIONS FOR A THERMODYNAMICALLY CONSISTENT BECKER-DÖRING MODEL*
}

\author{
VINCENT SSEMAGANDA ${ }^{\dagger}$ AND GERALD WARNECKE ${ }^{\dagger}$
}

\begin{abstract}
We study a thermodynamically consistent Becker-Döring model introduced by Dreyer and Duderstadt [J. Stat. Phys., 123, No. 1 (2006)]. In this model the fluxes have a possible singularity. We consider a more general model which accounts for the presence of an inert substance in a given system. We prove existence and uniqueness of solutions by using standard methods both for the original singular case and the extension to inert substances. Our results show that due to the structure of the model, solutions avoid the singularity if appropriate initial conditions are considered. We also study existence of equilibrium solutions. In some cases there is an upper bound on the mass contained in an equilibrium solution.
\end{abstract}

Key words. Nucleation, Becker-Döring model, kinetics of phase transition.

AMS subject classifications. 34A12, 34A34.

1. Introduction. Becker and Döring [2] introduced the so-called Becker-Döring model in 1935. The model considers finite or infinite systems of particle clusters. Particles are called molecules and their number at time $t$ is denoted as $z_{1}(t)$, whereas clusters consist of two or more molecules. Denote the number of clusters containing $i$ molecules at time $t$ by $z_{i}(t)$. In this model the size of a cluster containing $i$ molecules changes by either gaining a molecule at a condensation rate $C_{i}$ or by losing a molecule at an evaporation rate $E_{i}$. We introduce the flux $J_{i}(\mathbf{z}(t))$ as the net rate of conversion of clusters with $i$ molecules into those with $(i+1)$. It is given by

$$
J_{i}(\mathbf{z}(t))=C_{i} z_{i}(t)-E_{i+1} z_{i+1}(t) .
$$

The Becker-Döring system for $\mathbf{z}(t):=\left\{z_{i}(t)\right\}_{1 \leq i \leq \infty}$ is written as

$$
\begin{aligned}
& \dot{z}_{1}(t)=-J_{1}(\mathbf{z}(t))-\sum_{i=1}^{\infty} J_{i}(\mathbf{z}(t)), \\
& \dot{z}_{i}(t)=J_{i-1}(\mathbf{z}(t))-J_{i}(\mathbf{z}(t)) \text { for } i \geq 2,
\end{aligned}
$$

with initial conditions

$$
z_{i}(0)=z_{0 i} \geq 0, \quad i \geq 1
$$

The model is fully defined if the expressions for $C_{i}$ and $E_{i}$ in (1.1) are given.

We study the model introduced by Dreyer and Duderstadt [4] in 2006. They derived the so-called thermodynamically consistent Becker-Döring model by making use of the second law of thermodynamics. They considered two systems. One concerns the formation of liquid droplets in vapor while the other involves nucleation of liquid droplets in crystalline Gallium Arsenide surrounded by an inert gas. Suppose $N_{D}$ denotes the total number of clusters and free molecules in the system. Then we have

$$
N_{D}(\mathbf{z}(t)):=\sum_{i=1}^{\infty} z_{i}(t) .
$$

\footnotetext{
*Received June 13, 2012; accepted for publication July 4, 2013.

$\dagger^{\dagger}$. Ssemaganda is the corresponding author. Institute for Analysis and Numerics, Otto-vonGuericke University Magdeburg, Universitätsplatz 2, D-39106 Magdeburg, Germany (\{vincent. ssemaganda; gerald.warnecke\}@ovgu.de).
} 
Let $\mathcal{A}_{i}$ denote the availability of a system containing a single cluster of $i$ molecules surrounded by free molecules. With the convention $\mathcal{A}_{1}=0$, Dreyer and Duderstadt [4] expressed the total availability $\mathcal{A}$ for a many cluster system by

$$
\mathcal{A}(\mathbf{z}(t))=\sum_{i=1}^{\infty} \mathcal{A}_{i} z_{i}(t)+\beta \sum_{i=1}^{\infty} z_{i}(t) \ln \left(\frac{z_{i}(t)}{N_{D}(t)}\right) .
$$

The first term on the right-hand side is a sum of the availabilities of single cluster systems. The second term takes care of the entropy of mixing. We give three examples of systems where the expressions defining $\mathcal{A}_{i}$ are known.

EXAMPLE 1.1. This corresponds to a simple liquid-vapor system in which a spherical liquid droplet containing $i$ molecules is surrounded by its vapor. At at a temperature $T$ and outer pressure $p_{0}$ the availability $\mathcal{A}_{i}$ is given by, see Müller and Müller [9, pp. 304]

$$
\mathcal{A}_{1}=0, \quad \mathcal{A}_{i}=-\beta i \ln \left(\frac{p_{0}}{\bar{p}(T)}\right)+\gamma i^{2 / 3}, \quad i \geq 2, \quad \beta, \gamma>0
$$

where $\bar{p}(T)$ is the vapor-liquid equilibrium pressure at the temperature $T$.

EXAMPLE 1.2. This concerns a liquid solid crystallization system in which a spherical crystal germ containing $i$ molecules is surrounded by a liquid. Let $\Delta T$ denote the supercooling of the liquid. Then the availability is given by, see Kelton [7, Equations (3.3) and (9.4)]

$$
\mathcal{A}_{1}=0, \quad \mathcal{A}_{i}=-\eta i \Delta T+\gamma i^{2 / 3}, \quad i \geq 2, \quad \eta, \gamma>0 .
$$

EXAMPLE 1.3. Here the system consists of a single liquid droplet of size $i$ contained in a crystalline solid. Both are a binary mixture of gallium and arsenic. The solid is surrounded by an inert gas with prescribed pressure. For large $i$, the availability grows linearly with $i$, see Herrmann et al. [6]. This leads to the ansatz

$$
\mathcal{A}_{i}=\beta i, \quad \text { for } \quad i \gg 1, \quad \beta>0 .
$$

By using the condition $\dot{\mathcal{A}}_{i}(t) \leq 0$, which is the second law of thermodynamics, Dreyer and Duderstadt [4] derived a time dependent relationship between the condensation and evaporation rates as

$$
E_{i+1}(t)=C_{i} \frac{N_{D}(\mathbf{z}(t)) q_{i}}{z_{1}(t) q_{i+1}}, \quad i \geq 1
$$

where $\beta>0$ and $q_{i}:=\exp \left(-\mathcal{A}_{i} / \beta\right)$ do not depend on time for all $i \geq 1$. This relationship between the rates shows that if the condensation rates $C_{i}$ are specified, then the evaporation rates can be determined accordingly.

Dreyer and Duderstadt [4] derived condensation rates of the form

$$
C_{i}=4 \pi r_{i}^{2} \frac{z_{1}(t)}{V_{v}(t)} \sqrt{\frac{k T}{2 \pi m}}
$$


involving the radius $r_{i}$ of an $i$-molecule cluster, the volume $V_{v}(t)$ of vapor, the Boltzmann constant $k$, the temperature $T$ and the molecular mass $m$. They then used the ideal gas law in the form

$$
\frac{z_{1}(t)}{V_{v}(t)}=\frac{p_{0}}{k T}
$$

using the constant outer pressure $p_{0}$. This leads to the size dependent constant condensation rate

$$
C_{i}=4 \pi r_{i}^{2} \frac{p_{0}}{\sqrt{2 \pi m k T}}=: a_{i} .
$$

Now substituting (1.9) and (1.10) into (1.1) gives

$$
J_{i}(\mathbf{z}(t))=a_{i}\left(z_{i}(t)-\frac{q_{i}}{q_{i+1}} \frac{N_{D}(\mathbf{z}(t))}{z_{1}(t)} z_{i+1}(t)\right), \quad i \geq 1 .
$$

These fluxes together with (1.2) constitute the thermodynamically consistent BeckerDöring model of Dreyer and Duderstadt [4]. We observe that the fluxes may be singular in $z_{1}(t)$.

Herrmann et al. [6] discussed some of the mathematical properties of the thermodynamically consistent model. Instead of the condensation rates in (1.10) the authors considered those of the form

$$
C_{i}=\gamma_{i} z_{1}(t)
$$

by arguing that thermodynamics dictates the relationship (1.9) but does not restrict the choice of the condensation rates. This form was also used with different notation by Ball et al. [1] and there the equivalent of $\gamma_{i}$ as well as evaporation rates were referred to as kinetic coefficients. Using such rates leads to non-singular fluxes but transforms the problem to a different time scale $\tau$, which we define here as, $\tau(t):=\int_{0}^{t} z_{1}(s) d s$. Herrmann et al. [6] studied existence and uniqueness of solutions to the transformed problem as well as their asymptotic behavior under some conditions on the kinetic coefficients $\gamma_{i}$ and functions $q_{i}$. In the end however, they did not construct solutions to the original, possibly singular, problem because they did not prove that they may reverse the above transformation. Following the time scale defined above one can construct solutions to the original problem for all times $t>0$ only if the following three conditions are satisfied

$$
z_{1}(t)>0 \text { for } t<\infty, \quad \tau(0)=0, \quad \text { and } \quad \tau(t) \rightarrow \infty \text { for } t \rightarrow \infty .
$$

The solutions constructed by Hermann et al. [6] satisfy the first two conditions. The third one requires existence of a uniform lower bound on $z_{1}(t)$ other than zero or knowledge of the decay of $z_{1}(t)$ as $t \rightarrow \infty$. In particular $z_{1}(t)$ must not go to zero faster than $1 / t$. Herrmann et al. [6] showed that under some conditions the solution $\mathbf{z}(t)$ tends to zero as $t \rightarrow \infty$. Unfortunately, they did not discuss the behavior of the decay of $z_{1}(t)$. For this reason we study existence and uniqueness of solutions to (1.2) for fluxes defined in (1.11). We avoid the above transformation and prove existence directly for the original problem.

Before we embark on this task, we first generalize the thermodynamically consistent model to consider nucleation in presence of inert substances. This was motivated 
by the fact that many applications such as fog formation as well as nucleation experiments include inert substances. For instance experiments by Brus et al. [3] as well as Wölk and Strey [14] on homogeneous nucleation of water vapor and Argon nucleation studied by Sinha et al. [12]. The physical importance of the inert gas is that only due to its presence a stable coexistence of both phases is possible. Without the inert gas the system will tend to a one phase equilibrium. This is seen by studying the thermodynamic availability, see for example Müller and Müller [9, Chapter 11]. Let $z_{0} \geq 0$ denote the number of inert molecules in the given system. Then the general thermodynamically consistent Becker-Döring model for $\mathbf{z}(t)$ is given by

$$
\begin{aligned}
& \dot{z}_{0}(t)=0 \\
& \dot{z}_{1}(t)=-J_{1}(\mathbf{z}(t))-\sum_{i=1}^{\infty} J_{i}(\mathbf{z}(t)), \\
& \dot{z}_{i}(t)=J_{i-1}(\mathbf{z}(t))-J_{i}(\mathbf{z}(t)) \quad \text { for } \quad i \geq 2,
\end{aligned}
$$

with initial conditions

$$
z_{0}(0)=z_{0} \geq 0, \quad z_{i}(0)=z_{0 i} \geq 0, \quad i \geq 1 .
$$

In [13] we considered nucleation of liquid droplets in a vapor-inert gas mixture. We used the kinetic theory of gases to derive the condensation rates for this case. If the temperature and total pressure of the system are constant, the rates are given by

$$
C_{i}=a_{i} \frac{z_{1}(t)}{z_{0}+z_{1}(t)}
$$

where the constants $a_{i}$ are those used in (1.10). We also showed in [13] that the presence of an inert substance changes the entropy of mixing. Therefore for $z_{0}>0$ the summation in the second term on the right-hand side of (1.5) starts from zero. By using the second law of thermodynamics, as was done by Dreyer and Duderstadt [4], we were able to show in [13] that relationship (1.9) still holds even in the presence of an inert substance in the given system. In this case however, the total number $N_{D}(\mathbf{z}(t))$ of clusters and free molecules at time $t$ is defined as

$$
N_{D}(\mathbf{z}(t))=z_{0}+\sum_{i=1}^{\infty} z_{i}(t) .
$$

Therefore with this definition and the condensation rates in (1.16) the fluxes in (1.1) become

$$
J_{i}(\mathbf{z}(t))=a_{i}\left(z_{i}(t) \frac{z_{1}(t)}{z_{0}+z_{1}(t)}-\frac{q_{i}}{q_{i+1}} \frac{N_{D}(\mathbf{z}(t))}{z_{0}+z_{1}(t)} z_{i+1}(t)\right) .
$$

The important point to note here is that the reciprocal $\left(z_{0}+z_{1}\right)^{-1}$ appears in the flux (1.18). Therefore we have a finite or infinite system of ordinary differential equations with a possible singularity in the right-hand side. It must be shown that under appropriate initial conditions this singularity is avoided by the solutions. It is clear that there is no singularity in the fluxes if $z_{0}>0$.

Suppose that $m_{0}$ and $m_{1}$ are the molecular masses of the two substances in the given system. Then the total mass $\rho(t)$ is given by

$$
\rho(t)=m_{0} z_{0}+m_{1} \sum_{i=1}^{\infty} i z_{i}(t) .
$$


For mathematical convenience we set $m_{0}=m_{1}=1$ so that the total mass becomes

$$
\rho(t)=z_{0}+\sum_{i=1}^{\infty} i z_{i}(t)
$$

We show by Assumption 3.1 that the quotient $N_{D}(t) /\left(z_{0}+z_{1}(t)\right)$ is bounded uniformly for all $t \in[0, \infty[$. This bound shows that if appropriate initial conditions are chosen, the solutions will avoid the singularity at all times, $t \in[0, \infty[$. Existence of solutions is given in Theorem 3.9 which is proved using the standard methods discussed by Ball et al. [1]. To prove uniqueness of solutions, we distinguish between the case for $z_{0}>0$ and that for $z_{0}=0$. In general, for $C_{i}$ from (1.16), uniqueness of solutions for $z_{0}=0$ is only possible if the coefficients $a_{i}$ are size independent. This is stated in Theorem 4.4 (ii). However, by making a stronger restriction on the initial data, we are able to prove uniqueness locally for the whole class of $a_{i}$ given by (3.1), see Remark 4.5. We point out that only for $z_{0}=0$ and $C_{i}$ given by (1.12) did Hermann et al. [6] prove existence and uniqueness for the whole class of $a_{i}$ discussed in this paper.

For $z_{0}>0$ equilibrium solutions exist under more general conditions than those for which $z_{0}=0$. In some cases there exists an upper bound on the mass contained in an equilibrium solution, see (5.8). This is similar to the behavior of solutions for the standard mass conserving Becker-Döring model which was discussed by Ball et al. [1]. It is an interesting question to investigate the asymptotic behavior of solutions under the different conditions given in Theorem 5.2. We believe that the long time results for $z_{0}=0$, discussed by Herrmann et al. [6] can easily be adopted to answer this question. Therefore we do not discuss the long time dynamics in this paper. Our work is organized in the following way.

In Section 2 we introduce the appropriate functional spaces. We state some of the results which are essential for the following sections. Section 3 contains existence of solutions. We discuss uniqueness of solutions for the two cases $z_{0}>0$ and $z_{0}=0$ in Section 4. Finally, in Section 5 we discuss existence of equilibria for the model.

2. Function spaces. In this section we introduce the appropriate function spaces and state some results which are essential for the next sections. We are interested in solutions for which the total mass remains finite for all time. Let $\mathbb{N}_{0}$ denote the set of natural numbers including zero, that is, $\mathbb{N}_{0}:=\mathbb{N} \bigcup\{0\}$. Like in Ball et al. [1] we introduce the Banach sequence space

$$
\mathbf{X}=\left\{\mathbf{z}=\left(z_{i}\right)_{i \in \mathbb{N}_{0}} \subseteq \mathbb{R}:\|\mathbf{z}\|<\infty\right\}, \quad \text { where } \quad\|\mathbf{z}\|=\left|z_{0}\right|+\sum_{i=1}^{\infty} i\left|z_{i}\right| .
$$

We write $\mathbf{z} \geq 0$ if $z_{i} \geq 0$ for all $i \in \mathbb{N}_{0}$. We choose $\rho \geq 0$ and set

$$
\mathbf{X}^{+}=\{\mathbf{z} \in \mathbf{X}: \mathbf{z} \geq 0\} \quad \text { and } \quad \mathbf{X}_{\rho}^{+}=\left\{\mathbf{z} \in \mathbf{X}^{+}:\|\mathbf{z}\|=\rho\right\}
$$

Theorem 2.1. The Banach space $\mathbf{X}$ can be identified with the dual space $\mathbf{Y}^{*}$ of the space

$$
\mathbf{Y}=\left\{\mathbf{y}=\left(y_{i}\right)_{i \in \mathbb{N}_{0}} \subseteq \mathbb{R}: \lim _{i \rightarrow \infty} \frac{y_{i}}{i}=0\right\}, \quad \text { with norm } \quad\|\mathbf{y}\|_{\mathbf{Y}}=\max _{i \geq 1}\left\{\left|y_{0}\right|, \frac{\left|y_{i}\right|}{i}\right\} .
$$


The duality pairing of $\mathbf{X}$ and $\mathbf{Y}$ is defined as

$$
\langle\mathbf{y}, \mathbf{z}\rangle=\sum_{i=0}^{\infty} z_{i} y_{i}
$$

The proof of this theorem can be done similarly to the results by Naldzhieva [10] by making the transformation $z_{i} \rightarrow z_{i+1}$ and noting that

$$
\|\mathbf{z}\|_{\mathbf{x}}=\left|z_{1}\right|+\sum_{i=2}^{\infty}(i-1)\left|z_{i}\right| \quad \text { and } \quad\|\mathbf{z}\|_{\mathbf{Y}}=\max _{i \geq 2}\left\{\left|y_{1}\right|, \frac{\left|y_{i}\right|}{i-1}\right\}
$$

Lemma 2.2 (Ball, Carr and Penrose). A sequence $\mathbf{z}^{(n)}$ of elements of $\mathbf{X}$ converges weak ${ }^{*}$ to $\mathbf{z} \in \mathbf{X}$, we write $\mathbf{z}^{(n)} \rightarrow^{*} \mathbf{z}$, if and only if

(i) $z_{i}^{(n)} \rightarrow z_{i}$ as $n \rightarrow \infty$ for each $i \in \mathbb{N}_{0}$ and

(ii) $\sup _{n \in \mathbb{N}_{0}}\left\|\mathbf{z}^{(n)}\right\|_{\mathbf{x}}<\infty$.

The proof can be constructed by using the transformation $z_{i} \rightarrow z_{i+1}$ and the norms in (2.5). We can easily adapt the results in Naldzhieva [10] to construct the proof.

Definition 2.3 (Ball, Carr and Penrose). Let $\mathbf{E} \subset \mathbf{X}$. A function $\theta: \mathbf{E} \rightarrow \mathbb{R}$ is sequentially weak ${ }^{*}$ continuous if for $\mathbf{z}^{(n)} \in \mathbf{E}$ and $\mathbf{z} \in \mathbf{E}$ with $\mathbf{z}^{(n)} \rightarrow^{*} \mathbf{z}, \theta\left(\mathbf{z}^{(n)}\right) \longrightarrow^{*}$ $\theta(\mathbf{z})$.

Lemma 2.4 (Ball, Carr and Penrose). For $\mathbf{z} \in \mathbf{X}^{+}$and $\mathbf{y} \in \mathbf{Y}$ the functional $B(\mathbf{z})$ defined by $B(\mathbf{z}):=\sum_{i=0}^{\infty} y_{i} z_{i}$ is well defined and sequentially weak ${ }^{*}$ continuous. In particular

$$
N_{D}(\mathbf{z})=\sum_{i=0}^{\infty} z_{i}
$$

is sequentially weak* continuous.

The proof of Lemma 2.4 follows directly from the results in Lemma 2.2 and the fact that the constant sequence $(1,1, \ldots) \in \mathbf{Y}$.

3. Existence of solutions. Here we prove existence of solutions to the model for the general case $z_{0} \geq 0$. We consider the problem where the condensation rates $C_{i}$ are given by (1.16). This form of the rates is the one introduced by Dreyer and Duderstadt [4] for $z_{0}=0$. Let us make the following assumptions.

Assumption 3.1. For all $i \geq 1$ and $\Delta_{i}:=q_{i} / q_{i+1}>0$ we have the conditions:

(i) There are constants $\alpha \in[0,1[$ and $\xi>0$ such that

$$
a_{i}=\xi i^{\alpha} .
$$

(ii) $\frac{\Delta_{i+1} a_{i+1}}{a_{i+2}} \leq \frac{\Delta_{i} a_{i}}{a_{i+1}}$ and $0<R:=\lim _{i \rightarrow \infty} \Delta_{i}<\infty$. This implies that

$$
0<\kappa:=\inf _{i \geq 1}\left\{\frac{\Delta_{i} a_{i}}{a_{i+1}}, 1\right\}=\min \{R, 1\} \quad \text { and } \quad 0<\nu:=\sup _{i \geq 1} \Delta_{i}<\infty
$$


(iii) The initial data $\mathbf{z}(0) \in \mathbf{X}^{+}$satisfy $z_{0}+z_{1}(0)>0$.

With $b_{i+1}:=\Delta_{i} a_{i}$ the first inequality in Assumption 3.1 (ii) is equivalent to the condition $b_{i+1} / a_{i+1} \leq b_{i} / a_{i}$ used by Penrose [11, pp. 518]. The constant $R$ can be identified with the fugacity of saturated vapor. Therefore the requirement $\kappa>0$ in (3.2) is a reasonable one. We will use it later to show that (3.22) holds.

For $z_{0}=0$ Assumption 3.1 (iii) is physically reasonable for nucleation of condensed phases since free molecules must exist in the old phase before nucleation can take place. As a consequence, this assumption implies that the initial total mass $\rho(0)=$ $\sum_{i=1}^{\infty} i z_{i}(0)$ in the system is strictly positive.

REMARK 3.2. We point out that one could use the assumption $a_{i}=O(i)$ which was used by Ball et al. [1] as well as Herrmann et al. [6]. Although this gives a bigger class of kinetic coefficients, we are not aware of any applications with rates other than those of the form in (3.1). In fact, with this assumption we are able to prove existence of solutions in a stronger sense by establishing the continuity of the series in (3.42).

Definition 3.3. A function $\mathbf{z}:\left[0, T\left[\rightarrow \mathbf{X}^{+}\right.\right.$is a solution of $(1.14)$ on $[0, T[$, $0 \leq T \leq \infty$ if for given initial data $\mathbf{z}(0) \in \mathbf{X}^{+}$with $z_{0}(0)+z_{1}(0)>0$ the following conditions are satisfied:

(i) $\mathbf{z} \geq 0$ on $[0, T[$.

(ii) $\sup _{t \in[0, T[}\|\mathbf{z}(t)\|<\infty$, where $\|\mathbf{z}(t)\|:=z_{0}+\sum_{i=1}^{\infty} i z_{i}(t)$.

(iii) z solves (1.14), that is, each $z_{i}:[0, T[\rightarrow \mathbb{R}$ is differentiable.

It is convenient to divide the space $\mathbf{X}^{+}$into two disjoint subspaces $\mathbf{A}$ and $\mathbf{B}$ which are chosen such that

$$
\mathbf{A}:=\left\{\mathbf{z} \in \mathbf{X}^{+}: z_{0}+z_{1}<\frac{\kappa}{2} \sum_{i=2}^{\infty} z_{i}\right\} \quad \text { and } \quad \mathbf{B}:=\left\{\mathbf{z} \in \mathbf{X}^{+}: z_{0}+z_{1} \geq \frac{\kappa}{2} \sum_{i=2}^{\infty} z_{i}\right\}
$$

with $\kappa$ defined in (3.2). We have introduced the two sets above in order to make our arguments concerning existence of solutions mathematically clear. We have no physical reason to justify this distinction. The most important point is that the initial data must satisfy Assumption 3.1 (iii). Further, we will see that solutions starting in $\mathbf{A}$ will move to $\mathbf{B}$ whereas those originating in $\mathbf{B}$ will remain there. We analyze existence and uniqueness properties of solutions whose initial data are taken from either of the subspaces above. The solution is constructed in three steps. First, in Section 3.1 we prove existence of solutions to the finite dimensional system of size $n \in \mathbb{N}$ obtained by using the truncation $J_{n}\left(\mathbf{z}^{(n)}\right)=0$ and $z_{i}=0$ for all $i \geq n+1$. The results are summarized in Lemmas 3.4 and 3.6. Next, in Section 3.2 we construct an admissible limiting sequence via Lemma 3.8. Finally, we show in Section 3.3 that the constructed admissible sequence is consistent with the infinite system. This is a standard procedure used by Ball et al. [1]. The existence result is given in Theorem 3.9. The most important novel point of the existence result is our ability to prove the bound (3.39), which shows that the solutions avoid the singularity in the fluxes for all values of $z_{0} \geq 0$.

3.1. Finite system. Here we prove existence of a unique solution $\mathbf{z}^{(n)}$ to the finite dimensional system of size $n \in \mathbb{N}$ obtained by using $J_{n}\left(\mathbf{z}^{(n)}\right)=0$ and $z_{i}=0$ for 
all $i \geq n+1$ in (1.14). It is given as

$$
\begin{aligned}
\dot{z}_{0} & =0 \\
\dot{z}_{1}^{(n)}(t) & =-J_{1}\left(\mathbf{z}^{(n)}(t)\right)-\sum_{i=1}^{n-1} J_{i}\left(\mathbf{z}^{(n)}(t)\right), \\
\dot{z}_{i}^{(n)}(t) & =J_{i-1}\left(\mathbf{z}^{(n)}(t)\right)-J_{i}\left(\mathbf{z}^{(n)}(t)\right) \quad \text { for } \quad 2 \leq i \leq n-1, \\
\dot{z}_{n}^{(n)}(t) & =J_{n-1}\left(\mathbf{z}^{(n)}(t)\right) .
\end{aligned}
$$

The system is solved for initial data obtained as truncations of the data $\mathbf{z}(0) \in \mathbf{X}^{+}$ satisfying Assumption 3.1 (iii). We consider the initial conditions

$$
z_{i}^{(n)}(0)=z_{i}(0) \quad \text { for } \quad 0 \leq i \leq n .
$$

Further, we introduce $\rho^{(n)}(t):=z_{0}+\sum_{i=1}^{n} i z_{i}^{(n)}(t)$ and note that $\rho^{(n)}(0)>0$. In system (3.4), the total number of clusters and free molecules is given by $N_{D}^{(n)}(t):=$ $\sum_{i=0}^{n} z_{i}^{(n)}(t)$, while the fluxes are obtained from (1.18) as

$$
J_{i}\left(\mathbf{z}^{(n)}(t)\right)=a_{i}\left(\frac{z_{1}^{(n)}(t)}{z_{0}+z_{1}^{(n)}(t)} z_{i}^{(n)}(t)-\Delta_{i} \frac{N_{D}^{(n)}(t)}{z_{0}+z_{1}^{(n)}(t)} z_{i+1}^{(n)}(t)\right) .
$$

Now suppose that the initial data $\mathbf{z}(0)$ belong to the subspace $\mathbf{B}$ defined in (3.3). Then (1.15) and (3.5) mean that

$$
z_{0}+z_{1}^{(n)}(0) \geq \frac{\kappa}{2} \sum_{i=2}^{n} z_{i}^{(n)}(0)
$$

Define $f^{(n)}(t):=z_{0}+z_{1}^{(n)}(t)-\kappa / 2 \sum_{i=2}^{n} z_{i}^{(n)}(t)$. Then (3.7) implies that $f^{(n)}(0) \geq 0$. By using (3.4) we have

$$
\begin{aligned}
\dot{f}^{(n)}(t) & =\dot{z}_{1}^{(n)}(t)-\frac{\kappa}{2} \sum_{i=2}^{n} \dot{z}_{i}^{(n)}(t) \\
& =-J_{1}\left(\mathbf{z}^{(n)}(t)\right)-\sum_{i=1}^{n-1} J_{i}\left(\mathbf{z}^{(n)}(t)\right)-\frac{\kappa}{2} J_{1}\left(\mathbf{z}^{(n)}(t)\right) .
\end{aligned}
$$

Lemma 3.4. The system (3.4) - (3.6) with initial data in $\mathbf{B}$ has a unique solution for $t \geq 0$. At every $t$ the solution satisfies the conditions $z_{0}+z_{1}(t)>0, z_{i}(t) \geq 0$ for all $2 \leq i \leq n$ and $f^{(n)}(t) \geq 0$. Furthermore, the mass in the system is conserved, that $i s, \rho^{(\bar{n})}(t)=z_{0}+\sum_{i=1}^{n} i z_{i}(t)=z_{0}+\sum_{i=1}^{n} i z_{i}(0)$.

Proof. For $\varepsilon>0$ consider the following system obtained by adding $\varepsilon$ to each of the right-hand side terms of (3.4) for $2 \leq i \leq n$ and denote the resulting solution by $\mathbf{z}^{(\varepsilon)}$. Then

$$
\dot{z}_{i}^{\varepsilon}(t)=\left\{\begin{array}{lc}
J_{i-1}\left(\mathbf{z}^{(\varepsilon)}(t)\right)-J_{i}\left(\mathbf{z}^{(\varepsilon)}(t)\right)+\varepsilon ; & \text { for } \quad 2 \leq i \leq n-1, \\
J_{n-1}\left(\mathbf{z}^{(\varepsilon)}(t)\right)+\varepsilon, & \text { for } \quad i=n,
\end{array}\right.
$$


with initial conditions

$$
z_{i}^{(\varepsilon)}(0):=z_{i}^{(n)}(0)+\varepsilon>0, \quad 2 \leq i \leq n .
$$

In order to satisfy (3.7) the initial number of free molecules is transformed into

$$
z_{1}^{(\varepsilon)}(0)=z_{1}^{(n)}(0)+\varepsilon+\frac{\kappa}{2}(n-1) \varepsilon>0 .
$$

We modify the free molecule equation in (3.4) in the following way

$$
\dot{z}_{1}^{(\varepsilon)}(t)=-J_{1}\left(\mathbf{z}^{(\varepsilon)}(t)\right)-\sum_{i=1}^{n-1} J_{i}\left(\mathbf{z}^{(\varepsilon)}(t)\right)+\varepsilon+\frac{\kappa}{2}(n-1) \varepsilon .
$$

We will show later that this choice of modification is chosen so that the solution stays in the set $\mathbf{B}$. We have the mass

$$
\rho^{(\varepsilon)}(0)=\rho^{(n)}(0)+\left(\frac{n(n+1)}{2}+\frac{\kappa(n-1)}{2}\right) \varepsilon>0 .
$$

Note that for this system the mass increases linearly in time. To see this we differentiate $\rho^{(\varepsilon)}$ with respect to $t$ and use (3.9) together with (3.12) to obtain

$$
\begin{aligned}
\frac{d}{d t} \rho^{(\varepsilon)}(t)= & \sum_{i=1}^{n} i \dot{z}_{i}^{(\varepsilon)}(t), \\
= & -J_{1}\left(\mathbf{z}^{(\varepsilon)}(t)\right)-\sum_{i=1}^{n-1} J_{i}\left(\mathbf{z}^{(\varepsilon)}(t)\right)+\varepsilon+\frac{\kappa}{2}(n-1) \varepsilon \\
& +\sum_{i=2}^{n} i\left(J_{i-1}\left(\mathbf{z}^{(\varepsilon)}(t)\right)-J_{i}\left(\mathbf{z}^{(\varepsilon)}(t)\right)+\varepsilon\right), \\
= & \varepsilon\left(1+\frac{\kappa}{2}(n-1)+\sum_{i=2}^{n} i\right)>0 .
\end{aligned}
$$

With $f^{(\varepsilon)}(t):=z_{0}+z_{1}^{(\varepsilon)}(t)-\kappa / 2 \sum_{i=2}^{n} z_{i}^{(\varepsilon)}(t)$, the analogous equation to (3.8) becomes

$$
\dot{f}^{(\varepsilon)}(t)=-J_{1}\left(\mathbf{z}^{(\varepsilon)}(t)\right)-\sum_{i=1}^{n-1} J_{i}\left(\mathbf{z}^{(\varepsilon)}(t)\right)-\frac{\kappa}{2} J_{1}\left(\mathbf{z}^{(\varepsilon)}(t)\right)+\varepsilon .
$$

We note from the definition of the fluxes that the right-hand sides of each of (3.9) and (3.12) are continuous functions of $\mathbf{z}^{(\varepsilon)}$ for $z_{0}+z_{1}^{(\varepsilon)}(t)>0$. Moreover they are even continuously differentiable functions if $z_{0}+z_{1}^{(\varepsilon)}(t)>0$. Since the mass in the system is increasing we may deduce from $f^{(\varepsilon)}(t) \geq 0$ that $z_{0}+z_{1}^{(\varepsilon)}(t)>0$. Suppose $\tau_{\varepsilon}$ is the first time at which $f^{(\varepsilon)}\left(\tau_{\varepsilon}\right)=0$. Then $z_{0}+z_{1}^{(\varepsilon)}(t)>0$ on $[0, t]$ for any $\left.\left.t \in\right] 0, \tau_{\varepsilon}\right]$ so that the right-hand sides of (3.9) and (3.12) are locally Lipschitz continuous with respect to $\mathbf{z}^{(\varepsilon)}$ on the interval $[0, t]$. The Lipschitz constant can be constructed from the Jacobian matrix of the right-hand side functions as the maximum value of its rows. Thus local existence of a solution is guaranteed by using the Picard-Lindelöf theorem.

To prove positivity of this solution we note that the initial conditions (3.10) and (3.11) are strictly positive for $1 \leq i \leq n$. Moreover by assumption, the inequality 
$f^{(\varepsilon)}(t)>0$ holds on the interval $\left[0, \tau_{\varepsilon}\right.$ [ of existence. Suppose $\left.s \in\right] 0, \tau_{\varepsilon}$ [ is the first time for which one of the components $z_{i}^{(\varepsilon)}$ becomes zero. That is, there exists an $m$, $1 \leq m \leq n$, such that $z_{m}^{(\varepsilon)}(s)=0$. At $s$ we must have

$$
\dot{z}_{m}^{(\varepsilon)}(s) \leq 0
$$

and

$$
z_{i}^{(\varepsilon)}(s) \geq 0, \quad \text { for all } 1 \leq i \leq n
$$

After substituting (3.6) for the fluxes $J_{i}$ in (3.9) we have (3.16)

$$
\dot{z}_{m}^{\varepsilon}(s)=\left\{\begin{array}{l}
2 a_{1} \Delta_{1} \frac{N_{D}(s)}{z_{0}} z_{2}^{(\varepsilon)}(s)+\sum_{i=2}^{n-1} a_{i} \Delta_{i} \frac{N_{D}(s)}{z_{0}} z_{i+1}^{(\varepsilon)}(s)+\varepsilon+\frac{\kappa}{2}(n-1) \varepsilon, \text { if } m=1 \text { and } z_{0} \neq 0, \\
a_{m-1} \frac{z_{1}^{(\varepsilon)}(s)}{z_{0}+z_{1}^{(\varepsilon)}(s)} z_{m-1}^{(\varepsilon)}(s)+a_{m} \Delta_{m} \frac{N_{D}(s)}{z_{0}+z_{1}^{(\varepsilon)}(s)} z_{m+1}^{(\varepsilon)}(s)+\varepsilon ; \quad \text { if } 2 \leq m \leq n-1, \\
a_{n-1} \frac{z_{1}^{(\varepsilon)}(s)}{z_{0}+z_{1}^{(\varepsilon)}(s)} z_{n-1}^{(\varepsilon)}(s)+\varepsilon \quad \text { if } \quad m=n .
\end{array}\right.
$$

Thus $\dot{z}_{m}^{n}(s)>0$ for all $1 \leq m \leq n$. This is a contradiction to the inequality in (3.15) and therefore proves that $z_{i}^{(\varepsilon)}(\cdot)>0$ on $[0, t]$ for any $\left.t \in\right] 0, \tau_{\varepsilon}[$ and all $1 \leq i \leq n$. Note that if $z_{0}=0$ we must exclude the case for $m=1$ in $(3.16)$ since $f^{(\varepsilon)}(s)>0$ implies that $z_{1}^{(\varepsilon)}(s)$ cannot become zero.

Next we show that the solution can be extended to the whole interval $\left[0, \tau_{\varepsilon}\right]$ by proving that it is bounded up to $\tau_{\varepsilon}<\infty$. For this we integrate (3.13) to obtain

$$
0<z_{i}^{(\varepsilon)}(t) \leq z_{0}+\sum_{i=1}^{n} i z_{i}^{(\varepsilon)}(t)=z_{0}+\sum_{i=1}^{n} i z_{i}^{(\varepsilon)}(0)+t \varepsilon\left(1+\frac{\kappa}{2}(n-1)+\sum_{i=2}^{n} i\right)
$$

Thereby the solution is bounded for all $\left.t \in] 0, \tau_{\varepsilon}\right]$.

Finally to prove global existence we show that for the above $\tau_{\varepsilon}$ we have $\tau_{\varepsilon}=\infty$. Suppose that $\tau_{\varepsilon}<\infty$. Then due to the condition $f^{(\varepsilon)}(0)>0$ and continuity of $f^{(\varepsilon)}(t)$ we must have

$$
\dot{f}^{(\varepsilon)}\left(\tau_{\varepsilon}\right) \leq 0
$$

and

$$
z_{i}^{(\varepsilon)}\left(\tau_{\varepsilon}\right) \geq 0, \quad \text { for all } \quad 0 \leq i \leq n .
$$

Now we substitute for the fluxes according to (3.6) into (3.14) and use $z_{i}^{(\varepsilon)}(t)>0$ for 
all $1 \leq i \leq n$ on $] 0, \tau_{\varepsilon}[$ to obtain

$$
\begin{aligned}
\dot{f}^{(\varepsilon)}(t)= & \varepsilon-\left(2+\frac{\kappa}{2}\right) a_{1} \frac{z_{1}^{(\varepsilon)}}{z_{0}+z_{1}^{(\varepsilon)}} z_{1}^{(\varepsilon)}+\left(1+\frac{\kappa}{2}\right) \frac{N_{D}}{z_{0}+z_{1}^{(\varepsilon)}} z_{2}^{(\varepsilon)} a_{1} \Delta_{1}+a_{n} \frac{z_{1}^{(\varepsilon)}}{z_{0}+z_{1}^{(\varepsilon)}} z_{n}^{(\varepsilon)} \\
& +\sum_{i=2}^{n}\left(\frac{N_{D}}{z_{0}+z_{1}^{(\varepsilon)}} \frac{a_{i-1} \Delta_{i-1}}{a_{i}}-\frac{z_{1}^{(\varepsilon)}}{z_{0}+z_{1}^{(\varepsilon)}}\right) a_{i} z_{i}^{(\varepsilon)}, \\
> & \varepsilon-\left(2+\frac{\kappa}{2}\right) a_{1} \frac{z_{1}^{(\varepsilon)}}{z_{0}+z_{1}^{(\varepsilon)}} z_{1}^{(\varepsilon)}+\sum_{i=2}^{n}\left(\frac{N_{D}}{z_{0}+z_{1}^{(\varepsilon)}} \kappa-\frac{z_{1}^{(\varepsilon)}}{z_{0}+z_{1}^{(\varepsilon)}}\right) a_{i} z_{i}^{(\varepsilon)},
\end{aligned}
$$

$$
>\varepsilon-\left(2+\frac{\kappa}{2}\right) a_{1} z_{1}^{(\varepsilon)}+\sum_{i=2}^{n}\left(\frac{N_{D}}{z_{0}+z_{1}^{(\varepsilon)}} \kappa-1\right) a_{i} z_{i}^{(\varepsilon)} .
$$

We note that

$$
\frac{N_{D}^{(\varepsilon)}(t)}{z_{0}+z_{1}^{(\varepsilon)}(t)}=1+\frac{1}{z_{0}+z_{1}^{(\varepsilon)}(t)} \sum_{i=2}^{n} z_{i}^{(\varepsilon)}(t)
$$

Using this relation and $f^{(\varepsilon)}\left(\tau_{\varepsilon}\right)=z_{0}+z_{1}^{(\varepsilon)}\left(\tau_{\varepsilon}\right)-\kappa / 2 \sum_{i=2}^{n} z_{i}^{(\varepsilon)}\left(\tau_{\varepsilon}\right)=0$ we have

$$
\dot{f}^{(\varepsilon)}\left(\tau_{\varepsilon}\right)>\varepsilon-\frac{\kappa}{2}\left(2+\frac{\kappa}{2}\right) a_{1} \sum_{i=2}^{n} z_{i}^{(n)}\left(\tau_{\varepsilon}\right)+(1+\kappa) \sum_{i=2}^{n} a_{i} z_{i}^{(n)}\left(\tau_{\varepsilon}\right) .
$$

Next we use the condition $a_{i}>a_{1}$ on the condensation rates and rearrange the results to obtain

$$
\dot{f}^{(\varepsilon)}\left(\tau_{\varepsilon}\right)>\varepsilon+a_{1}\left(1-\frac{\kappa^{2}}{4}\right) \sum_{i=2}^{n} z_{i}^{(n)}\left(\tau_{\varepsilon}\right) .
$$

Since $\kappa \leq 1$, the above inequality implies that $\dot{f}^{(\varepsilon)}\left(\tau_{\varepsilon}\right)>0$. This contradicts (3.18) for $\tau_{\varepsilon}<\infty$. Therefore $\tau_{\varepsilon}=\infty$ and the solution exists for all $t \in[0, \infty[$ due to the bound in (3.17). To conclude the proof of Lemma 3.4 we take the limit $\varepsilon \rightarrow 0$ to obtain $\mathbf{z}^{(n)}(t)=\lim _{\varepsilon \rightarrow 0} \mathbf{z}^{(\varepsilon)}(t) \geq 0$. We note that the above convergence is uniform, see Hartmann [5, pp. 5 and 25]. In particular, the condition $f^{(n)}(t) \geq 0$ is satisfied. Mass conservation is obtained by taking $\varepsilon=0$ in (3.13). This leads to the following uniform upper bound on the solution

$$
z_{i}^{(n)}(t) \leq i^{-1} \rho^{(n)}(0)
$$

for each $i \geq 1$.

REMARK 3.5. We point out that we can use the condition $f^{(n)}(t) \geq 0$ to obtain an upper bound on the quotient $N_{D}^{(n)} /\left(z_{0}+z_{1}^{(n)}\right)$ via

$$
\frac{N_{D}^{(n)}(t)}{z_{0}+z_{1}^{(n)}(t)} \leq 1+\frac{2}{\kappa} \text {. }
$$

Note that if $z_{0}>0$ the denominator on the left-hand side of the above inequality is bounded from below. It can easily be shown that $N_{D}^{(n)} /\left(z_{0}+z_{1}^{(n)}\right) \leq \rho^{(n)}(0) / z_{0}$. 
However, for $z_{0}=0$ the condition (3.24) does not explicitly give a time independent lower bound on the number $z_{1}^{(n)}(t)$ of free molecules. To construct a lower bound on $z_{1}^{n}(t)$ we note that the mass satisfies the bound

$$
\rho^{n}(t)=\sum_{i=1}^{n} i z_{i}(t) \leq n \sum_{i=1}^{n} z_{i}(t)=n N_{D}^{(n)}(t) .
$$

Thus by using mass conservation we have

$$
N_{D}^{(n)}(t) \geq \frac{\rho^{(n)}(0)}{n}
$$

It follows from (3.24) and (3.25) that

$$
z_{1}^{(n)}(t) \geq \frac{\kappa}{(\kappa+2)} \frac{\rho^{(n)}(0)}{n} .
$$

Therefore (3.26) acts as a lower bound on the number of free molecules for a fixed $n$ on $[0, \infty[$.

Now we consider existence and uniqueness of solutions for the finite system (3.4) with initial data taken from A. The result is summarized in Lemma 3.6.

Lemma 3.6. The system (3.4) - (3.6) with initial data in $\mathbf{A}$ has a unique solution for all $t \geq 0$ with $z_{0}+z_{1}(t)>0$ and $z_{i}(t) \geq 0$ for $1 \leq i \leq n$. There exists a finite time $t_{\mathbf{A}}$ such that the time derivative $\dot{z}_{1}^{(n)}(t)$ of $z_{1}^{(n)}(t)$ satisfies $\dot{z}_{1}^{(n)}(t)>0$ on $\left[0, t_{\mathbf{A}}\right]$. In particular, $t_{\mathbf{A}}$ is the time it takes for the solution to enter region $\mathbf{B}$ from $\mathbf{A}$. Furthermore, the mass in the system is conserved, i.e. $\rho^{(n)}(t)=\rho^{(n)}(0)$.

Proof. Existence, uniqueness and mass conservation can be proved analogously to the results in Lemma 3.4. For existence of the time $t_{\mathbf{A}}$ it is sufficient to show that if $\mathbf{z}^{(n)}(t) \in \mathbf{A}$ at time $t$, then $\dot{z}_{1}^{(n)}(t)>0$. Consider the free molecule equation in (3.4) and substitute the fluxes according to (3.6). Then following the same procedure as in the derivation of $(3.20)$ we obtain

$$
\dot{z}_{1}^{(n)}(t)>-2 a_{1} z_{1}^{(n)}+\sum_{i=2}^{n}\left(\frac{N_{D}^{(n)}}{z_{0}+z_{1}^{(n)}} \kappa-1\right) a_{i} z_{i}^{(n)},
$$

or equivalently

$$
\dot{z}_{1}^{(n)}(t)>-2 a_{1} z_{1}^{(n)}+\sum_{i=2}^{n}\left(\kappa+\frac{\sum_{i=2}^{n} z_{i}^{(n)}}{z_{0}+z_{1}^{(n)}} \kappa-1\right) a_{i} z_{i}^{(n)} .
$$

Recall that $\mathbf{z}^{(n)}(t) \in \mathbf{A}$ implies that $z_{0}+z_{1}^{(n)}(t)<\kappa / 2 \sum_{i=2}^{n} z_{i}^{(n)}$. Now using this condition together with $a_{i}>a_{1}$ for all $2 \leq i \leq n$ we obtain

$$
\dot{z}_{1}^{(n)}(t)>\frac{2}{\kappa} a_{1}\left(z_{0}+z_{1}^{(n)}(t)\right) .
$$

The assumption $z_{0}+z_{1}^{(n)}(0)>0$ implies that the right-hand side of the above inequality is greater than zero for all $t \in\left[0, t_{\mathbf{A}}\right]$. To prove the finiteness of $t_{\mathbf{A}}$ we note that (3.27) implies that

$$
z_{1}^{(n)}(t)>z_{1}^{(n)}(0) \exp \left(\frac{2}{\kappa} a_{1} t\right)
$$


The conservation of mass means that after a finite time $t_{m}$, with $t_{m}<$ $\left(\kappa / 2 a_{1}\right) \ln \left(\rho^{(n)}(0) / z_{1}^{(n)}(0)\right)$, all the mass will be contained in the free molecules. It is clear that at $t_{m}$ the solution will already be in region $\mathbf{B}$. The proof ends by noting that continuity of time implies that there exists a time $t_{\mathbf{A}}$ with $t_{\mathbf{A}}<t_{m}$.

REMARK 3.7. The above result is important for the proof of uniqueness of solutions if $z_{0}=0$, see Remark 4.5. In this case Assumption 3.1 tells us that $z_{1}(0)>0$. On $\left[0, t_{\mathbf{A}}\right]$ the value of $z_{1}^{(n)}(t)$ is bounded below by $z_{1}^{(n)}(0)$. Thus we can use mass conservation to bound the quotient $N_{D}^{(n)} / z_{1}^{(n)}$ by

$$
\frac{N_{D}^{(n)}(t)}{z_{1}^{(n)}(t)} \leq \frac{\rho^{(n)}(0)}{z_{1}^{(n)}(0)}
$$

on $\left[0, t_{\mathbf{A}}\right]$.

3.2. Admissible solution. In the second step we construct an admissible solution which is a limit of a sub-sequence $\mathbf{z}^{\left(n_{k}\right)}(\cdot)$ of solutions $\mathbf{z}^{(n)}(\cdot)$ as $k \rightarrow \infty$. To construct the admissible solution we apply the Arzela-Ascoli theorem by showing that for each $1 \leq i \leq n$ the sequence of solutions $z_{i}^{(n)}$ is uniformly bounded and equicontinuous. Uniform boundedness follows directly from (3.23) while for equicontinuity it is sufficient to show that the derivatives $\dot{z}_{i}^{(n)}(t)$ are uniformly bounded in $i$ and $t$. We use the bounds (3.24) and (3.28) to define a constant $K$ by

$$
K:=\max \left\{1+\frac{2}{\kappa}, \frac{\rho}{z_{1}(0)}\right\}
$$

so that for all initial conditions taken from $\mathbf{X}^{+}$the solution satisfies

$$
\frac{N_{D}^{(n)}}{z_{0}+z_{1}^{n}} \leq K
$$

From (3.4) we have

$$
\left|\dot{z}_{i}^{(n)}(t)\right| \leq\left\{\begin{array}{l}
\left|J_{i-1}\left(\mathbf{z}^{(n)}(t)\right)\right|+\left|J_{i}\left(\mathbf{z}^{(n)}(t)\right)\right| ; \quad \text { if } \quad 2 \leq i \leq n \\
\left|J_{1}\left(\mathbf{z}^{(n)}(t)\right)\right|+\left|\sum_{i=1}^{n} J_{i}\left(\mathbf{z}^{(n)}(t)\right)\right| \quad \text { if } \quad i=1 .
\end{array}\right.
$$

Using Assumption 3.1 (i) the fluxes satisfy

$$
\begin{aligned}
\left|J_{i}\left(\mathbf{z}^{(n)}(t)\right)\right| & \leq \sum_{i=1}^{n}\left|J_{i}\left(\mathbf{z}^{(n)}(t)\right)\right| \\
& \leq \xi \sum_{i=1}^{n}\left(i^{\alpha} z_{i}^{(n)}(t)+i^{\alpha} z_{i+1}^{(n)}(t) \frac{N_{D}^{(n)}(t)}{z_{0}+z_{1}^{(n)}(t)} \Delta_{i}\right)
\end{aligned}
$$

Next we use the conditions $\alpha \in\left[0,1\left[, \nu=\sup _{i \geq 1} \Delta_{i}<\infty\right.\right.$, and (3.30) together with the mass-conservation property for finite systems to obtain

$$
\left|J_{i}\left(\mathbf{z}^{(n)}(t)\right)\right| \leq \sum_{i=1}^{n}\left|J_{i}\left(\mathbf{z}^{(n)}(t)\right)\right| \leq(1+K \nu) \xi \rho^{(n)}(0) .
$$


Hence we have

$$
\left|\dot{z}_{i}^{(n)}(t)\right| \leq 2(1+K \nu) \xi \rho^{n}(0)
$$

for all $1 \leq i \leq n$ and for all $t \in\left[0, \infty\right.$ [. If the mass $\rho^{(n)}(0)$ is bounded independently of $n$ then $z_{i}^{(n)}(t)$ and $\dot{z}_{i}^{(n)}(t)$ are uniformly bounded in $n, i$ and $t$, giving the equicontinuity of the sequence.

Lemma 3.8 (Admissible solution for $z_{0}=0$ ). Let $\mathbf{z}(0)=\left(z_{0 i}\right)_{i \in \mathbb{N}_{0}} \in \mathbf{X}_{\rho}^{+}$with $z_{0}+z_{01}>0$. Now for every $n \in \mathbb{N}$ let $\mathbf{z}^{(n)} \in C\left(\left[0, \infty\left[; \mathbb{R}^{n}\right)\right.\right.$ be the unique solution of (3.4) for the initial data defined by

$$
z_{0 i}^{(n)}=\left\{\begin{array}{l}
\frac{\rho}{z_{0}+\sum_{k=1}^{n} k z_{0 k}} z_{0 i} ; \quad \text { for } \quad 1 \leq i \leq n, \\
0 \quad \text { otherwise. }
\end{array}\right.
$$

Then there exists a sub-sequence $\left(\mathbf{z}^{\left(n_{k}\right)}\right)_{k \in \mathbb{N}}$ of $\left(\mathbf{z}^{(n)}\right)_{n \in \mathbb{N}}$ and a function $\mathbf{z}:[0, \infty[\rightarrow \mathbf{X}$ such that

(i) For every $i \in \mathbb{N}_{0}, z_{i}$ is continuous and $z_{i}^{\left(n_{k}\right)} \stackrel{k \rightarrow \infty}{\longrightarrow} z_{i}$ uniformly on compact subsets of $[0, \infty[$.

(ii) The total number $N_{D}(\mathbf{z})$ of clusters and free molecules is continuous and $N_{D}\left(\mathbf{z}^{\left(n_{k}\right)}\right) \stackrel{k \rightarrow \infty}{\longrightarrow} N_{D}(\mathbf{z})$ uniformly on compact subsets of $[0, \infty[$.

Proof. We note that the choice (3.34) of initial conditions implies that the mass $\rho^{(n)}(0)$ is such that $\rho^{(n)}(0)=\rho$ for all $n \in \mathbb{N}$. Therefore from (3.23) and (3.33) the sequence $z_{i}^{(n)}(t)$ is uniformly bounded and equicontinuous for each $i \in \mathbb{N}_{0}$. Thus by the Arzela Ascoli theorem there exists a sub-sequence $z_{1}^{\left(n_{k}\right)}$ and a function $z_{1}$ such that $z_{1}^{\left(n_{k}\right)}$ converges uniformly to $z_{1}$ on compact subsets of [0, $\infty$ [as $k \rightarrow \infty$. Similarly there exists a sub-sequence $z_{2}^{\left(n_{k}\right)}$ converging uniformly to $z_{2}$ on compact subsets of $\left[0, \infty\left[\right.\right.$ as $k \rightarrow \infty$. This procedure is repeated for $z_{3}^{(n)}, z_{4}^{(n)}, \ldots$ and so on. By using the diagonalization method we can extract a sequence $\mathbf{z}^{\left(n_{k}\right)}$ so that $z_{i}^{\left(n_{k}\right)} \stackrel{k \rightarrow \infty}{\longrightarrow} z_{i}$ uniformly for every $i \in \mathbb{N}_{0}$ on compact subsets of $\left[0, \infty\left[\right.\right.$. Since for every $n_{k}$, we have $z_{i}^{n_{k}} \in C\left(\left[0, \infty[)\right.\right.$ and $z_{i}^{n_{k}}(t) \geq 0$, then due to uniform convergence we must have $z_{i} \in C\left(\left[0, \infty[)\right.\right.$ and $z_{i}(t) \geq 0$ for all $t \in[0, \infty[$.

Furthermore since $N_{D}$ is weak* continuous due to Lemma 2.4, then $N_{D}\left(\mathbf{z}^{\left(n_{k}\right)}\right)$ converges pointwise in time to $N_{D}(\mathbf{z})$ for $k \rightarrow \infty$. Besides we have

$$
\left|\dot{N}_{D}\left(\mathbf{z}^{\left(n_{k}\right)}\right)\right|=\left|-\sum_{i=1}^{n_{k}} J_{i}\left(\mathbf{z}^{\left(n_{k}\right)}\right)\right| .
$$

Using (3.32) then gives

$$
\left|\dot{N}_{D}\left(\mathbf{z}^{\left(n_{k}\right)}\right)\right| \leq(1+K \nu) \xi \rho
$$

Similarly it is clear from mass conservation that $N_{D}\left(\mathbf{z}^{\left(n_{k}\right)}\right) \leq \rho$. Therefore by the Arzela Ascoli theorem there exists a sub-sequence still denoted by $\mathbf{z}^{\left(n_{k}\right)}$ such that the sequence $N_{D}\left(\mathbf{z}^{\left(n_{k}\right)}\right)$ converges to some $N_{D}$ uniformly on compact subsets of $[0, \infty[$. Due to uniqueness of the limit we must have $N_{D}(t)=N_{D}(\mathbf{z}(t))=\sum_{i=1}^{\infty} i z_{i}(t)$ for all $t \geq 0$. 
Up to this point we have constructed an admissible solution $\mathbf{z}$ to (1.14) via Lemma 3.8. In the following section we prove that this solution is consistent with the infinite system (1.14).

3.3. Consistency. Here we show that the admissible solution constructed above actually solves (1.14).

TheOrem 3.9 (Existence for $z_{0}=0$ ). Let the conditions of Lemma 3.8 be fulfilled. Then $\mathbf{z}$ is a solution of (1.14) on $\left[0, \infty\left[\right.\right.$ for initial data $\mathbf{z}(0)=\left(z_{0 i}\right)_{i \in \mathbb{N}_{0}}$. In particular we obtain $z_{i} \in C\left(\left[0, \infty[)\right.\right.$ for every $i \in \mathbb{N}_{0}$.

Proof. For the sub-sequence $\mathbf{z}^{\left(n_{k}\right)}$ we note by using (3.1) and (3.6) that

$$
\begin{aligned}
\lim _{k \rightarrow \infty} J_{i}\left(\mathbf{z}^{\left(n_{k}\right)}(t)\right) & =\lim _{k \rightarrow \infty} \xi i^{\alpha}\left(\frac{z_{1}^{\left(n_{k}\right)}(t)}{z_{0}+z_{1}^{\left(n_{k}\right)}(t)} z_{i}^{\left(n_{k}\right)}(t)-\Delta_{i} z_{i+1}^{\left(n_{k}\right)}(t) \frac{N_{D}\left(\mathbf{z}^{\left(n_{k}\right)}(t)\right)}{z_{0}+z_{1}^{\left(n_{k}\right)}(t)}\right) \\
& =\xi i^{\alpha}\left(\frac{z_{1}(t)}{z_{0}+z_{1}(t)} z_{i}(t)-\Delta_{i} z_{i+1}(t) \frac{N_{D}(\mathbf{z}(t))}{z_{0}+z_{1}(t)}\right) \\
& =J_{i}(\mathbf{z}(t)),
\end{aligned}
$$

with the convergence being uniform on compact subsets of $[0, \infty[$. Thus we have $J_{i}(\mathbf{z}) \in C\left(\left[0, \infty[)\right.\right.$. Since from (3.32) the flux $J_{i}\left(\mathbf{z}^{\left(n_{k}\right)}(t)\right)$ is uniformly bounded, using the Lebesgue dominated convergence theorem we observe that

$$
\lim _{k \rightarrow \infty} \int_{0}^{t} J_{i}\left(\mathbf{z}^{\left(n_{k}\right)}(s)\right) d s=\int_{0}^{t} J_{i}(\mathbf{z}(s)) d s .
$$

Besides, the continuity of $J_{i}(\mathbf{z})$ in time implies that the fundamental theorem of calculus can be applied to give

$$
\frac{d}{d t} \int_{0}^{t} J_{i}(\mathbf{z}(s)) d s=J_{i}(\mathbf{z}(t))
$$

The convergence of the term $\sum_{i=1}^{n_{k}} J_{i}\left(\mathbf{z}^{\left(n_{k}\right)}(t)\right)$ which appears in the differential equation for the free molecules is more involved. First of all we have to show that it is uniformly bounded and converges pointwise towards a finite limit $\sum_{i=1}^{\infty} J_{i}(\mathbf{z}(t))$ as $k \rightarrow \infty$. Secondly we must also show that the series $\sum_{i=1}^{\infty} J_{i}(\mathbf{z}(t))$ is uniformly convergent so as to guarantee its continuity. For each $n_{k}$ we regard the solution $\mathbf{z}^{n_{k}}$ to the finite system as an element of the space $\mathbf{X}$ defined in (2.1) by setting $z_{i}^{n_{k}}=0$ for all $i>n_{k}$. Then we can use the mass conservation property of these solutions to write

$$
\sum_{i=1}^{n_{k}} i z_{i}^{n_{k}}(t)=\sum_{i=1}^{\infty} i z_{i}^{n_{k}}(t)=\sum_{i=1}^{n_{k}} i z_{i}^{n_{k}}(0) \leq \sum_{i=1}^{\infty} i z_{i}(0)=\rho .
$$

Therefore, for a fixed $m \in \mathbb{N}$ we have

$$
\sum_{i=1}^{m-1} i z_{i}^{n_{k}}(t)+\sum_{i=m}^{\infty} i z_{i}^{n_{k}}(t) \leq \rho .
$$

By taking the limit $k \rightarrow \infty$ we note that both terms on the left-hand side of this inequality have finite limits, due to the bound on the right. In the first one we use 
$\lim _{k \rightarrow \infty} z_{i}^{n_{k}}(t)=z_{i}(t)$. Next we take the limit $m \rightarrow \infty$ to obtain

$$
\sum_{i=1}^{\infty} i z_{i}(t) \leq \rho
$$

It can easily be seen from (3.30) and the uniform convergence of both $N_{D}\left(\mathbf{z}^{n_{k}}(t)\right) \rightarrow$ $N_{D}(\mathbf{z}(t))$ and $z_{1}^{n_{k}}(t) \rightarrow z_{1}(t)$ for $k \rightarrow \infty$ that

$$
\frac{N_{D}(\mathbf{z}(t))}{z_{0}+z_{1}(t)} \leq K
$$

Now we use (3.38), (3.39) and Assumption 3.1 (ii), $\alpha<1$ to get

$$
\begin{aligned}
\left|\sum_{i=m}^{n} J_{i}(\mathbf{z}(t))\right| & \leq \sum_{i=m}^{n} \xi i^{\alpha}\left(z_{i}(t)+\Delta_{i} z_{i+1}(t) \frac{N_{D}(\mathbf{z}(t))}{z_{1}(t)}\right) \\
& \leq \sum_{i=m}^{n} \xi \frac{i}{i^{1-\alpha}}\left(z_{i}(t)+\Delta_{i} z_{i+1}(t) \frac{N_{D}(\mathbf{z}(t))}{z_{1}(t)}\right) \\
& \leq \frac{\xi}{m^{1-\alpha}}(1+K \nu) \rho .
\end{aligned}
$$

The bound is independent of $n$ and $t$. This implies that the series $\sum_{i=1}^{\infty} J_{i}(\mathbf{z}(t))$ is uniformly convergent. Continuity of the series follows from continuity of each $J_{i}(\mathbf{z})$. Analogously, for $m<n_{k}$, we can derive the following estimate for the finite system

$$
\left|\sum_{i=m}^{n_{k}} J_{i}\left(\mathbf{z}^{\left(n_{k}\right)}\right)\right| \leq \frac{\xi}{m^{1-\alpha}}(1+K \nu) \rho .
$$

This means that for each fixed $m<n_{k}$, the sequence $S_{m}^{n_{k}}:=\left|\sum_{i=m}^{n_{k}} J_{i}(\mathbf{z}(t))\right|$ is uniformly bounded and hence convergent. We can choose $m$ large enough to have

$$
\left|\sum_{i=m}^{n_{k}} J_{i}\left(\mathbf{z}^{\left(n_{k}\right)}\right)\right| \leq \frac{\varepsilon}{3} \quad \text { and } \quad\left|\sum_{i=m}^{\infty} J_{i}(\mathbf{z}(t))\right| \leq \frac{\varepsilon}{3} .
$$

Now these results together with the uniform convergence $z_{i}^{n_{k}}(t) \rightarrow z_{i}(t)$ lead to

$$
\begin{aligned}
\left|\sum_{i=1}^{n_{k}} J_{i}\left(\mathbf{z}^{\left(n_{k}\right)}(t)\right)-\sum_{i=1}^{\infty} J_{i}(\mathbf{z}(t))\right| \leq & \left|\sum_{i=1}^{m-1}\left(J_{i}\left(\mathbf{z}^{\left(n_{k}\right)}(t)\right)-J_{i}(\mathbf{z}(t))\right)\right|+\left|\sum_{i=m}^{n_{k}} J_{i}\left(\mathbf{z}^{\left(n_{k}\right)}(t)\right)\right| \\
& +\left|\sum_{i=m}^{\infty} J_{i}(\mathbf{z}(t))\right| \leq \frac{\varepsilon}{3}+\frac{\varepsilon}{3}+\frac{\varepsilon}{3} .
\end{aligned}
$$

Therefore we get

$$
\lim _{k \rightarrow \infty} \sum_{i=1}^{n_{k}} J_{i}\left(\mathbf{z}^{\left(n_{k}\right)}\right)=\sum_{i=1}^{\infty} J_{i}(\mathbf{z})<\infty .
$$

By the Lebesgue dominated convergence theorem we have

$$
\lim _{k \rightarrow \infty} \int_{0}^{t} \sum_{i=1}^{n_{k}} J_{i}\left(\mathbf{z}^{\left(n_{k}\right)}(s)\right) d s=\int_{0}^{t} \sum_{i=1}^{\infty} J_{i}(\mathbf{z}(s)) d s .
$$


Then we can again apply the fundamental theorem of calculus to write

$$
\frac{d}{d t} \int_{0}^{t} \sum_{i=1}^{\infty} J_{i}(\mathbf{z}(s)) d s=\sum_{i=1}^{\infty} J_{i}(\mathbf{z}(t)) .
$$

Next we consider convergence for the initial data in the limit $k \rightarrow \infty$. This gives

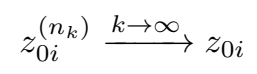

for $i \in \mathbb{N}$ because $\rho / \sum_{i=1}^{\left(n_{k}\right)} z_{0 i} \stackrel{k \rightarrow \infty}{\longrightarrow} 1$. Finally we write (3.4) in integral form as

$$
\begin{aligned}
& z_{1}^{\left(n_{k}\right)}(t)=z_{1}^{\left(n_{k}\right)}(0)-\int_{0}^{t} J_{1}\left(\mathbf{z}^{\left(n_{k}\right)}(s)\right) d s-\int_{0}^{t} \sum_{i=1}^{n_{k}-1} J_{i}\left(\mathbf{z}^{\left(n_{k}\right)}(s)\right) d s \\
& z_{i}^{\left(n_{k}\right)}(t)=z_{i}^{\left(n_{k}\right)}(0)+\int_{0}^{t} J_{i-1}\left(\mathbf{z}^{\left(n_{k}\right)}(s)\right) d s-\int_{0}^{t} J_{i}\left(\mathbf{z}^{\left(n_{k}\right)}(s)\right) d s \quad \text { for } \quad 2 \leq i \leq n \\
& z_{n}^{(n)}(t)=z_{n}^{(n)}(0)+\int_{0}^{t} J_{n_{k}-1}\left(\mathbf{z}^{\left(n_{k}\right)}(s)\right) d s
\end{aligned}
$$

Taking the limit as $k \rightarrow \infty$ in the above system and using (3.36), (3.41), and (3.43) we obtain

$$
\begin{aligned}
& z_{1}(t)=z_{1}(0)-\int_{0}^{t} J_{1}(\mathbf{z}(s)) d s-\int_{0}^{t} \sum_{i=1}^{\infty} J_{i}(\mathbf{z}(s)) d s \\
& z_{i}(t)=z_{i}(0)+\int_{0}^{t} J_{i-1}(\mathbf{z}(s)) d s-\int_{0}^{t} J_{i}(\mathbf{z}(s)) d s \quad \text { for } \quad i \geq 2 .
\end{aligned}
$$

To conclude the proof we note that the right-hand sides of the above system are continuously differentiable in $t$. Thus the left-hand sides must also be continuously differentiable. We can then differentiate both sides with respect to $t$ and use (3.37) together with (3.42) to show that the solution $\mathbf{z}$ solves (1.14) in the strong sense.

REMARK 3.10. We point out that the solution which was constructed by Herrmann et al. [6, Equation (A2) and the remark on pp. 121] was weak in the sense that the continuity of the series in (3.42) could not be established for the class of condensation rates they considered. Although they considered a bigger class of rates, we are not aware of any applications with rates other than those of the form in (3.1).

4. Uniqueness of solutions and Mass conservation. In this section we prove uniqueness of the solution by following an idea introduced by Laurençot and Mischler [8]. There, the authors assumed that the coefficients $a_{i}$ satisfy

$$
0 \leq a_{i} \quad \text { and } \quad a_{i+1}-a_{i} \leq K
$$

for some positive constant $K$. Clearly the choice $a_{i}=\xi i^{\alpha}, \alpha \in[0,1$ [, made in Section 3 satisfies this condition with $K=\xi$. In addition, we assume that

$$
M:=\sup _{i \geq 1}\left(a_{i} \Delta_{i}-a_{i+1} \Delta_{i+1}\right)<\infty .
$$

For $b_{i+1}=\Delta_{i} a_{i}$ this requirement is equivalent to to the condition $b_{i}-b_{i+1} \leq M$ which was used by Laurençot and Mischler [8, Eq. (2.3)]. 
We consider the case where the condensation rates are given by (1.16). Let $\mathbf{z}$ be a solution of (1.14) in the sense of Definition 3.3. Define a sequence $\mathbf{F}=\left(F_{i}\right)_{i \in \mathbb{N}_{0}}$ by

$$
F_{i}(t):=\sum_{j=i}^{\infty} z_{j}(t)
$$

Then $\mathbf{F}$ is a solution to the system

$$
\begin{aligned}
& \dot{F}_{i}(t)=J_{i-1}(\mathbf{F}(t)), \quad i \geq 2, \\
& \dot{F}_{0}(t)=\dot{F}_{1}(t)=-\sum_{i=1}^{\infty} J_{i}(\mathbf{F}(t)),
\end{aligned}
$$

where the fluxes are derived from (1.18) by using the substitution $z_{i}=F_{i}-F_{i+1}$ for $i \geq 0$. They are given by

$$
J_{i}(\mathbf{F}(t))=\xi i^{\alpha}\left[\left(F_{i}(t)-F_{i+1}(t)\right) \frac{F_{1}(t)-F_{2}(t)}{F_{0}(t)-F_{2}(t)}-\frac{\Delta_{i} F_{0}(t)}{F_{0}(t)-F_{2}(t)}\left(F_{i+1}(t)-F_{i+2}(t)\right)\right] .
$$

Definition 4.1. A function $\mathbf{F}:\left[0, T\left[\rightarrow l^{1}(\mathbb{R})\right.\right.$ is a solution of $(4.3)-(4.5)$ on $[0, T[, 0<T \leq \infty$ if

(i) $F_{i}(t) \geq F_{i+1}(t) \geq 0$ on $\left[0, T\left[\right.\right.$ for all $i \in \mathbb{N}_{0}$.

(ii) $\sup _{t \in[0, T[} \sum_{i=1}^{\infty} i\left(F_{i}(t)-F_{i+1}(t)\right)<\infty$.

(iii) $\lim _{n \rightarrow \infty} n F_{n+1}(t)=0$ for all $t \in[0, T[$.

(iv) $\mathbf{F}$ solves (4.3) - (4.5).

Claim. If $\mathbf{z}$ solves (1.14) then $\mathbf{F}$ solves (4.3) - (4.5). On the other hand if $\mathbf{F}$ solves (4.3) - (4.5) then the sequence $\mathbf{z}=\left(z_{i}\right)_{i \in \mathbb{N}_{0}}$ defined by $z_{i}=F_{i}-F_{i+1}$ solves (1.14). The proof of this claim can be done analogously to the results in Naldzhieva [10, Section 4]. For the first part, the idea is to write (1.14) as a system of integral equations and then use (4.2) with infinity replaced by a finite value, say $n$. The next step is to take the limit $n \rightarrow \infty$, noting that due to (3.40) $J_{n} \rightarrow 0$ in this limit. For the second part one has to first show existence of solutions for (4.3) - (4.5) by following the same procedure used in Section 3. Then with $z_{i}=F_{i}-F_{i+1}$ the solution to (1.14) can be constructed.

Lemma 4.2. Let $\mathbf{F}$ be a solution of (4.3) - (4.5). Then $\sum_{i=1}^{\infty} F_{i}(t)=\sum_{i=1}^{\infty} F_{i}(0)$ for all $t \geq 0$.

Proof. With $z_{i}=F_{i}-F_{i+1}$ we note that

$$
\sum_{i=1}^{n} i z_{i}(t)=\sum_{i=1}^{n} i\left(F_{i}(t)-F_{i+1}(t)\right)=\sum_{i=1}^{n} F_{i}(t)-n F_{n+1}(t) .
$$

Next we take the limit $n \rightarrow \infty$, while using Definition 4.1 (iii) and Definition 3.3 (ii) to obtain

$$
\sum_{i=1}^{\infty} F_{i}(t)=\sum_{i=1}^{\infty} i z_{i}(t)<\infty
$$

For $n \geq 2$ we have

$$
\sum_{i=2}^{n} F_{i}(t)-\sum_{i=2}^{n} F_{i}(0)=\int_{0}^{t} \sum_{i=2}^{n} J_{i-1}(\mathbf{F}(s)) d s=\int_{0}^{t} \sum_{i=1}^{n} J_{i}(\mathbf{F}(s)) d s .
$$


Next we take the limit as $n \rightarrow \infty$ while noting that $\sum_{i=1}^{\infty} J_{i}(\mathbf{F}(s))$ is convergent due to $\left|J_{i}(\mathbf{F}(t))\right| \leq$ const $\cdot i\left(F_{i}(t)-F_{i+1}(t)\right)$ and Definition 4.1 (ii). We therefore get the following result from (4.4)

$$
\sum_{i=2}^{\infty} F_{i}(t)-\sum_{i=2}^{\infty} F_{i}(0)=\int_{0}^{t} \sum_{i=1}^{\infty} J_{i}(\mathbf{F}(s)) d s=-F_{1}(t)+F_{1}(0) .
$$

The proof is accomplished by collecting like terms.

REMARK 4.3. Mass conservation of solutions for (1.14) is therefore equivalent to the conservation property for (4.3) and (4.4) proved in Lemma 4.2, since $\rho(t)=$ $z_{0}+\sum_{i=1}^{\infty} i z_{i}(t)=z_{0}+\sum_{i=1}^{\infty} F_{i}(t)$.

ThEOREM 4.4 (Uniqueness). Let $\mathbf{F}$ and $\hat{\mathbf{F}}$ be two solutions for (4.3) - (4.5) such that $\mathbf{F}(0)=\hat{\mathbf{F}}(0)$ for the initial data taken from $\mathbf{X}^{+}$.

(i) Suppose that $z_{0}>0$ and $\alpha \in[0,1[$,

or

(ii) Suppose that $z_{0}=0$ and $\alpha=0$,

Then we have $\mathbf{F}(t)=\hat{\mathbf{F}}(t)$ for all $t \geq 0$.

Proof. For the two solutions we have corresponding solutions $\mathbf{z}$ and $\hat{\mathbf{z}}$ to (1.14). Define $\mathbf{E}:=\mathbf{F}-\hat{\mathbf{F}}$. Then for $i \geq 2$ we get by (4.5)

$$
\begin{aligned}
\frac{d}{d t} E_{i}(t)= & \frac{d}{d t} F_{i}(t)-\frac{d}{d t} \hat{F}_{i}(t)=J_{i-1}(\mathbf{F}(t))-J_{i-1}(\hat{\mathbf{F}}(t)), \\
= & \xi(i-1)^{\alpha}\left[\frac{z_{1}\left(F_{i-1}-F_{i}\right)}{z_{0}+z_{1}}-\frac{\Delta_{i-1} F_{0}\left(F_{i}-F_{i+1}\right)}{z_{0}+z_{1}}\right. \\
& \left.-\frac{\hat{z}_{1}\left(\hat{F}_{i-1}-\hat{F}_{i}\right)}{z_{0}+\hat{z}_{1}}+\frac{\Delta_{i-1} \hat{F}_{0}\left(\hat{F}_{i}-\hat{F}_{i+1}\right)}{z_{0}+\hat{z}_{1}}\right] .
\end{aligned}
$$

We add and subtract the term $\xi(i-1)^{\alpha}\left[\left(\hat{F}_{i-1}-\hat{F}_{i}\right) z_{1} /\left(z_{0}+z_{1}\right)+\Delta_{i-1}\left(\hat{F}_{i}-\right.\right.$ $\left.\left.\hat{F}_{i+1}\right) F_{0} /\left(z_{0}+z_{1}\right)\right]$ to obtain

$$
\begin{aligned}
\frac{d}{d t} E_{i}(t)= & \xi(i-1)^{\alpha}\left[\frac{z_{1}\left(E_{i-1}-E_{i}\right)}{z_{0}+z_{1}}-\frac{\Delta_{i-1} F_{0}\left(E_{i}-E_{i+1}\right)}{z_{0}+z_{1}}\right. \\
& \left.+\left(\hat{F}_{i-1}-\hat{F}_{i}\right)\left(\frac{z_{1}}{z_{0}+z_{1}}-\frac{\hat{z}_{1}}{z_{0}+\hat{z}_{1}}\right)+\Delta_{i-1}\left(\hat{F}_{i}-\hat{F}_{i+1}\right)\left(\frac{\hat{F}_{0}}{z_{0}+\hat{z}_{1}}-\frac{F_{0}}{z_{0}+z_{1}}\right)\right]
\end{aligned}
$$

Next we add and subtract the term $\xi(i-1)^{\alpha}\left[\left(\hat{F}_{i-1}-\hat{F}_{i}\right) z_{1} /\left(z_{0}+\hat{z}_{1}\right)+\Delta_{i-1}\left(\hat{F}_{i}-\right.\right.$ $\left.\left.\hat{F}_{i+1}\right) F_{0} /\left(z_{0}+\hat{z}_{1}\right)\right]$ and then use $\hat{z}_{i}=\hat{F}_{i}-\hat{F}_{i+1}$. This gives

$$
\begin{aligned}
\frac{d}{d t} E_{i}(t)= & \xi(i-1)^{\alpha}\left[\frac{z_{1}\left(E_{i-1}-E_{i}\right)}{z_{0}+z_{1}}-\frac{\Delta_{i-1} F_{0}\left(E_{i}-E_{i+1}\right)}{z_{0}+z_{1}}\right. \\
& \left.+\frac{\hat{z}_{i-1}}{z_{0}+\hat{z}_{1}}\left(z_{1} \frac{\hat{z}_{1}-z_{1}}{z_{0}+z_{1}}+E_{1}-E_{2}\right)+\Delta_{i-1} \frac{\hat{z}_{i}}{z_{0}+\hat{z}_{1}}\left(\frac{F_{0}\left(z_{1}-\hat{z}_{1}\right)}{z_{0}+z_{1}}-E_{1}\right)\right] .
\end{aligned}
$$

We then apply $z_{1}-\hat{z}_{1}=\left(F_{1}-F_{2}\right)-\left(\hat{F}_{1}-\hat{F}_{2}\right)=E_{1}-E_{2}$ to get

$$
\begin{aligned}
\frac{d}{d t} E_{i}(t)= & \xi(i-1)^{\alpha}\left[\frac{z_{1}\left(E_{i-1}-E_{i}\right)}{z_{0}+z_{1}}-\frac{\Delta_{i-1} F_{0}\left(E_{i}-E_{i+1}\right)}{z_{0}+z_{1}}-\frac{\Delta_{i-1} \hat{z}_{i}}{z_{0}+\hat{z}_{1}} E_{1}\right. \\
& \left.+\left(\frac{\hat{z}_{i-1}}{z_{0}+\hat{z}_{1}}-\frac{\hat{z}_{i-1}}{z_{0}+\hat{z}_{1}} \frac{z_{1}}{z_{0}+z_{1}}+\frac{\Delta_{i-1} \hat{z}_{i}}{z_{0}+\hat{z}_{1}} \frac{F_{0}}{z_{0}+z_{1}}\right)\left(E_{1}-E_{2}\right)\right]
\end{aligned}
$$


Multiplying this equation by $\operatorname{sign}\left(E_{i}\right)$ and simplifying the result, we obtain

$$
\begin{aligned}
\frac{d}{d t}\left|E_{i}(t)\right| \leq & \xi(i-1)^{\alpha}\left[\frac{z_{1}\left(\left|E_{i-1}\right|-\left|E_{i}\right|\right)}{z_{0}+z_{1}}-\frac{\Delta_{i-1} F_{0}\left(\left|E_{i}\right|-\left|E_{i+1}\right|\right)}{z_{0}+z_{1}}\right. \\
& \left.+\left(\frac{z_{0} \hat{z}_{i-1}}{z_{0}+\hat{z}_{1}}+\frac{\Delta_{i-1} \hat{z}_{i} F_{0}}{z_{0}+\hat{z}_{1}}\right) \frac{\left|E_{2}\right|}{z_{0}+z_{1}}+\left(\frac{z_{0} \hat{z}_{i-1}}{z_{0}+z_{1}}+\frac{\Delta_{i-1} \hat{z}_{i} F_{2}}{z_{0}+z_{1}}\right) \frac{\left|E_{1}\right|}{z_{0}+\hat{z}_{1}}\right] .
\end{aligned}
$$

For $n \geq 3$ we sum this inequality from 2 to $n$ and simplify the result to

$$
\frac{d}{d t} \sum_{i=2}^{n}\left|E_{i}(t)\right| \leq \sum_{i=1}^{n+1} \beta_{i}\left|E_{i}\right| .
$$

The coefficients $\beta_{i}$ are now given by

$$
\beta_{i}=\left\{\begin{array}{l}
\frac{\xi z_{1}}{z_{0}+z_{1}}+\frac{\xi}{z_{0}+\hat{z}_{1}} \sum_{i=2}^{n}(i-1)^{\alpha}\left(\frac{z_{0} \hat{z}_{i-1}+\Delta_{i-1} \hat{z}_{i} F_{2}}{z_{0}+z_{1}}\right) \quad \text { for } \quad i=1, \\
\frac{\xi}{z_{0}+z_{1}}\left[2^{\alpha} z_{1}-\left(z_{1}+\Delta_{1} F_{0}\right)\right]+\frac{\xi}{z_{0}+\hat{z}_{1}} \sum_{i=2}^{n}(i-1)^{\alpha}\left(\frac{z_{0} \hat{z}_{i-1}+\Delta_{i-1} F_{0} \hat{z}_{i}}{z_{0}+z_{1}}\right) \quad \text { for } \quad i=2, \\
\frac{\xi z_{1}}{z_{0}+z_{1}}\left[i^{\alpha}-(i-1)^{\alpha}\right]+\frac{\xi F_{0}}{z_{0}+z_{1}}\left[(i-2)^{\alpha} \Delta_{i-2}-(i-1)^{\alpha} \Delta_{i-1}\right] \quad \text { for } \quad 3 \leq i \leq n-1, \\
\frac{\xi F_{0}}{z_{0}+z_{1}}\left[(n-2)^{\alpha} \Delta_{n-2}-(n-1)^{\alpha} \Delta_{n-1}\right]-\frac{\xi z_{1}}{z_{0}+z_{1}}(n-1)^{\alpha} \quad \text { for } \quad i=n, \\
\frac{\xi F_{0}}{z_{0}+z_{1}}(n-1)^{\alpha} \Delta_{n-1} \quad \text { for } i=n+1 .
\end{array}\right.
$$

Now we turn our attention to part (i) of the uniqueness theorem, the case where $z_{0}>0$. We show that the coefficients $\beta_{i}$ are bounded independently of $i$. We note that

$$
\frac{1}{z_{0}+\hat{z}_{1}} \leq \frac{1}{z_{0}}, \quad \frac{z_{1}}{z_{0}+z_{1}} \leq 1, \quad \text { and } \quad F_{i} \leq \rho, \quad \text { for } \quad i \geq 0 .
$$

By using the above inequalities, we can construct upper bounds for the coefficients $\beta_{1}$ and $\beta_{2}$ via

$$
\beta_{1} \leq \xi\left(1+\frac{z_{0} \hat{\rho}+\nu \hat{\rho} \rho}{z_{0}^{2}}\right) \quad \text { and } \quad \beta_{2} \leq \xi\left(1+\frac{z_{0} \hat{\rho}+z_{0} \nu \rho+\nu \hat{\rho} \rho}{z_{0}^{2}}\right),
$$

where $\nu$ is defined in Assumption 3.1 (ii) and $\rho, \hat{\rho}$ are the masses for the corresponding systems. We can also construct upper bounds for $\beta_{i}, 3 \leq i \leq n$, if the terms $\left[i^{\alpha}-\right.$ $\left.(i-1)^{\alpha}\right]$ and $\left[(i-2)^{\alpha} \Delta_{i-2}-(i-1)^{\alpha} \Delta_{i-1}\right]$ are bounded uniformly in $n$. This has already been done by Naldzhieva [10] for Example 1.3. For the other two examples, we use the condition $M<\infty$ in Assumption 3.1 (ii) and the monotonic decrease of $\left[i^{\alpha}-(i-1)^{\alpha}\right]$ with $i$. Finally we show that $\lim _{n \rightarrow 0} \beta_{n+1}\left|E_{n+1}(t)\right|=0$ for all $t \geq 0$. For this we have

$$
\begin{aligned}
\beta_{n+1}\left|E_{n+1}\right| & \leq \beta_{n+1}\left|F_{n+1}\right|+\beta_{n+1}\left|\hat{F}_{n+1}\right| \\
& \leq \beta_{n+1} \sum_{i=n+1}^{\infty} z_{i}+\beta_{n+1} \sum_{i=n+1}^{\infty} \hat{z}_{i} \\
& \leq \frac{\xi \nu \rho}{z_{0}} n^{\alpha} \sum_{i=n+1}^{\infty} i z_{i} / i+\frac{\xi \nu \rho}{z_{0}} n^{\alpha} \sum_{i=n+1}^{\infty} i \hat{z}_{i} / i \\
& \leq \frac{\xi \nu \rho}{z_{0}}\left(\frac{\rho+\hat{\rho}}{n^{1-\alpha}}\right) .
\end{aligned}
$$


The right-hand side goes to zero as $n \rightarrow \infty$. Equation (4.6) can be written in integral form as

$$
\sum_{i=2}^{n}\left|E_{i}(t)\right| \leq \sum_{i=2}^{n}\left|E_{i}(0)\right|+\int_{0}^{t}\left(\beta_{1}\left|E_{1}(s)\right|+\sum_{i=2}^{n+1} \beta_{i}\left|E_{i}(s)\right|\right) d s .
$$

Note that

$$
\sum_{i=1}^{\infty}\left|E_{i}(t)\right| \leq \sum_{i=1}^{\infty}\left|F_{i}(t)\right|+\sum_{i=1}^{\infty}\left|\hat{F}_{i}(t)\right|<\infty
$$

and

$$
\begin{aligned}
\left|E_{1}(t)\right| & =\left|F_{1}(t)-\hat{F}_{1}\right|=\left|\sum_{i=1}^{\infty} F_{i}(t)-\sum_{i=2}^{\infty} F_{i}(t)-\sum_{i=1}^{\infty} \hat{F}_{i}(t)+\sum_{i=2}^{\infty} \hat{F}_{i}(t)\right| \\
& \leq \sum_{i=2}^{\infty}\left|E_{i}(t)\right|+\sum_{i=1}^{\infty}\left|E_{i}(0)\right|,
\end{aligned}
$$

the last line being due to Lemma 4.2. Using (4.9) and (4.10) we can take the limit as $n \rightarrow \infty$ in (4.8) and apply the Lebesgue dominated convergence theorem to obtain

$$
\sum_{i=2}^{\infty}\left|E_{i}(t)\right| \leq\left(1+t \beta_{1}\right) \sum_{i=2}^{\infty}\left|E_{i}(0)\right|+2 \max _{i \geq 2} \beta_{i} \int_{0}^{t} \sum_{i=2}^{\infty}\left|E_{i}(s)\right| d s .
$$

Then the application of Gronwall's Lemma gives the desired result that $E_{i}(t)=0$ for all $i \geq 1$ and for all $t \geq 0$ and hence uniqueness.

For part (ii) we use $z_{0}=0$. The coefficients $\beta_{i}$ become

$$
\beta_{i}=\left\{\begin{array}{l}
\xi+\frac{F_{2}}{z_{1}} \sum_{i=2}^{n} \xi(i-1)^{\alpha} \Delta_{i-1} \frac{\hat{z}_{i}}{\hat{z}_{1}} \quad \text { for } i=1, \\
\xi\left[2^{\alpha}-\left(1+\Delta_{1} \frac{F_{1}}{z_{1}}\right)\right]+\left[\sum_{i=2}^{n} \xi(i-1)^{\alpha} \Delta_{i-1} \frac{\hat{z}_{i}}{\hat{z}_{1}}\right] \frac{F_{1}}{z_{1}} \quad \text { for } \quad i=2, \\
\xi\left[i^{\alpha}-(i-1)^{\alpha}\right]+\xi\left[(i-2)^{\alpha} \Delta_{i-2}-(i-1)^{\alpha} \Delta_{i-1}\right] \frac{F_{1}}{z_{1}} \quad \text { for } \quad 3 \leq i \leq n-1, \\
\xi\left[(n-2)^{\alpha} \Delta_{n-2}-(n-1)^{\alpha} \Delta_{n-1}\right] \frac{F_{1}}{z_{1}}-\xi(n-1)^{\alpha} \quad \text { for } \quad i=n, \\
\xi(n-1)^{\alpha} \Delta_{n-1} \frac{F_{1}}{z_{1}} \text { for } i=n+1 .
\end{array}\right.
$$

The bound (3.39) is equivalent to $F_{1} / z_{1} \leq K$, where $K$ is defined in (3.29). Since we have not constructed a time independent lower bound on $z_{1}(t)$ the coefficients $\beta_{1}$ and $\beta_{2}$ can only be bounded uniformly in $n$ if $\alpha=0$. Then we have

$$
\begin{aligned}
\beta_{1} & \leq \xi+\xi\left(F_{1} / z_{1}-1\right) \frac{\hat{F}_{2}}{\hat{z}_{1}} \nu \\
& \leq \xi[1+(K+1) K \nu] .
\end{aligned}
$$

Similarly, for $\beta_{2}$ we have

$$
\beta_{2} \leq \xi(1+K) K \nu
$$


For $3 \leq i \leq n-1$ we obtain

$$
\beta_{i} \leq 2 \xi K \nu
$$

while

$$
\beta_{n} \leq \xi(1+2 K \nu)
$$

The uniqueness result can be completed analogously to the proof of part (i).

REMARK 4.5. If it is known a priori that the initial data are taken from $\mathbf{A}$ then up to the time $t_{\mathbf{A}}$ introduced in Section 3 we have a lower bound on $z_{1}$ given by (3.28). We can therefore prove uniqueness locally for $\alpha \in\left[0,1\left[\right.\right.$ and $t \in\left[0, t_{\mathbf{A}}\right]$, since we saw in the proof of part (i) that the terms $\left[i^{\alpha}-(i-1)^{\alpha}\right]$ and $\left[(i-2)^{\alpha} \Delta_{i-2}-(i-1)^{\alpha} \Delta_{i-1}\right]$ are bounded uniformly in $n$.

5. Equilibrium distribution. An equilibrium state of the Becker-Döring system is a state $\overline{\mathbf{z}} \in \mathbf{X}^{+}$such that all fluxes $J_{i}$ vanish in $\overline{\mathbf{z}}$ and $\bar{z}_{0}=z_{0}$. In this section we discuss equilibrium solutions to (1.14) for the case where $z_{0}>0$. The case $z_{0}=0$ was discussed already by Herrmann et al. [6]. Define the following terms

$$
\tilde{q}_{i}:=R^{i} q_{i}, \quad \tilde{f}(\mu):=\sum_{i=1}^{\infty} \mu^{i} \tilde{q}_{i}, \quad \text { and } \quad \tilde{g}(\mu):=\sum_{i=1}^{\infty} i \mu^{i} \tilde{q}_{i} .
$$

Theorem 5.1 (Herrmann at al. [6]). Let $z_{0}=0$. For any given mass $\bar{\rho}>0$, there exists an equilibrium state $\overline{\mathbf{z}}$ with $\rho(\overline{\mathbf{z}})=\bar{\rho}$ if and only if

$$
\tilde{f}(1)>1, \quad \text { or } \quad \tilde{f}(1)=1 \quad \text { and } \quad \tilde{g}(1)<\infty .
$$

Moreover, if (5.2) is satisfied, then

(a) there exists a unique value $\bar{\mu} \in] 0,1]$ such that $\tilde{f}(\bar{\mu})=1$.

(b) the equilibrium solution is given by

$$
\bar{z}_{i}=N_{D}(\overline{\mathbf{z}}) \tilde{q}_{i} \bar{\mu}^{i}, \quad \text { for } \quad i \geq 1, \quad \text { and } \quad N_{D}(\overline{\mathbf{z}})=\frac{\bar{\rho}}{\tilde{g}(\bar{\mu})} .
$$

Now we turn our attention to the case where $z_{0}>0$. It is clear that $\mathbf{z}=$ $\left(z_{0}, 0,0, \ldots\right)$ is an equilibrium solution with mass $z_{0}$. We study equilibrium states with $\bar{z}_{0}=z_{0}$ and prescribed fixed positive total mass $\rho>z_{0}$. By setting the fluxes $J_{i}$ in (1.18) to zero, we obtain

$$
\bar{z}_{i+1}=\left(\frac{\bar{z}_{1}}{\bar{N}_{D}}\right) \frac{q_{i+1}}{q_{i}} \bar{z}_{i}=\left(\frac{\bar{z}_{1}}{R \bar{N}_{D}}\right) \frac{\tilde{q}_{i+1}}{\tilde{q}_{i}} \bar{z}_{i}, \quad i \geq 1 .
$$

With $\bar{\mu}:=\bar{z}_{1} /\left(R \bar{N}_{D}\right)$ this equation gives

$$
\bar{z}_{i}=\left(\frac{\bar{z}_{1}}{R \bar{N}_{D}}\right)^{i-1} \frac{\tilde{q}_{i}}{\tilde{q}_{1}} \bar{z}_{1}=\bar{N}_{D} \tilde{q}_{i} \bar{\mu}^{i}=\bar{N}_{D} q_{i}(R \bar{\mu})^{i}, \quad \text { for } \quad i \geq 1 .
$$

The condition $\bar{N}_{D}=N_{D}(\overline{\mathbf{z}})$ and the constraint $\rho(\overline{\mathbf{z}})=\bar{\rho}$ require that

$$
z_{0}+\bar{N}_{D} \tilde{f}(\bar{\mu})=\bar{N}_{D} \quad \text { and } \quad \bar{N}_{D} \tilde{g}(\bar{\mu})=\bar{\rho}-z_{0},
$$


where $\tilde{f}$ and $\tilde{g}$ are defined in (5.1). Note that both series have the same radius of convergence $\mu_{s}=1$ and are strictly increasing on $[0,1]$. We summarize the existence of equilibrium solutions in the following theorem.

TheOrem 5.2 (Equilibria). For any given mass $\rho$, with $0<\rho<\infty$, let $0 \leq$ $z_{0} /\left(\rho-z_{0}\right)<\infty$. Then there exists an equilibrium state $\overline{\mathbf{z}}$ with mass $\rho$ if and only if

$$
\tilde{f}(1)+\frac{z_{0} \tilde{g}(1)}{\rho-z_{0}} \geq 1 .
$$

Equivalently, this condition implies existence of equilibria if

$$
\tilde{f}(1) \geq 1 \quad \text { or } \quad \rho \leq z_{0}(1+\tilde{g}(1)),
$$

or if

$$
0<\frac{\tilde{g}(1)}{1-\tilde{f}(1)}<\infty \quad \text { and } \quad z_{0}(1+\tilde{g}(1))<\rho \leq z_{0}\left(1+\frac{\tilde{g}(1)}{1-\tilde{f}(1)}\right)
$$

Moreover, if (5.6) is satisfied then there exists a unique value $\bar{\mu}$ such that

$$
\rho=z_{0}\left(1+\frac{\tilde{g}(\bar{\mu})}{1-\tilde{f}(\bar{\mu})}\right) .
$$

The equilibrium solution is given by

$$
\bar{z}_{0}=z_{0}, \quad \bar{N}_{D}=\frac{\bar{\rho}-z_{0}}{\tilde{g}(\bar{\mu})}, \quad \text { and } \quad \bar{z}_{i}=\bar{N}_{D} \tilde{q}_{i} \bar{\mu}^{i} \quad \text { for } \quad i \geq 1 .
$$

This theorem shows that in the presence of an inert substance, there are more possibilities for the existence of equilibrium solutions than the condition (5.2) which was obtained by Herrmann et al. [6] for $z_{0}=0$. In fact one can obtain the results of Theorem 5.1 by setting $z_{0}=0$ in (5.6). The only case where equilibria of the form (5.10) do not exist is the negation of (5.8) given by

$$
0<\frac{\tilde{g}(1)}{1-\tilde{f}(1)}<\infty \quad \text { and } \quad z_{0}\left(1+\frac{\tilde{g}(1)}{1-\tilde{f}(1)}\right)<\rho .
$$

The second of these conditions means that

$$
\tilde{f}(1)+\frac{z_{0} \tilde{g}(1)}{\rho-z_{0}}<1
$$

which clearly contradicts the necessary and sufficient existence requirement (5.6).

We conclude this section by showing how the results in Theorem 5.2 apply to the examples given in the introduction. By defining $\bar{S}:=p_{0} / \bar{p}(T)$ or $\bar{S}:=\exp (\eta \Delta T / \beta)$, the two Examples 1.1 and 1.2 are equivalent. In both cases existence of an equilibrium state $\bar{z}$ with $z_{0}>0$ does not only depend on the values of $\bar{S}$ and $\gamma / \beta$, but also on the mass in the system. In particular, existence of equilibria fails for large values of $\bar{S}$, $\gamma / \beta$ and for a mass that satisfies (5.11). This is different from the case $z_{0}=0$ where existence of an equilibrium solution does not depend on the mass. In Example 1.3 the presence of an inert substance does not change the results discussed by Herrmann et al. [6]. In particular, we have $R=\exp (+\beta)>1$ so that the first inequality in (5.7) always holds. 
6. Conclusion. In the previous sections we discussed the existence and uniqueness of solutions for a thermodynamically consistent Becker-Döring model, with an inert substance represented by $z_{0} \geq 0$. For $z_{0}=0$ and evaporation rates of the form introduced by Dreyer and Duderstadt [4], we were able to prove a global uniqueness result only for size independent condensation rates. This is as a result of the failure to construct a time independent lower bound on $z_{1}(t)$ for all $t \geq 0$. In fact this is the same problem encountered when one is to prove existence and uniqueness for all $t \geq 0$ by using a time scaled problem, as in Herrmann et al. [6]. In particular, the time scale must satisfy the conditions in (1.13).

For $z_{0}>0$ we have proved existence and uniqueness for the whole class of condensation rates considered here. The conditions for existence of equilibrium solutions are more general than those for $z_{0}=0$, which were given by Herrmann et al. [6]. Under some conditions, there is an upper bound on the mass contained in an equilibrium solution. This is similar to the standard mass conserving Becker-Döring model which was studied by Ball et al. [1].

Acknowledgements. We gratefully acknowledge that the work of the authors was partially supported by funding of the State of Sachsen-Anhalt through the International Max Planck Research School (IMPRS) for Analysis, Design and Optimization in Chemical and Biochemical Process Engineering.

\section{REFERENCES}

[1] J. M. Ball, J. Carr, And O. Penrose, The Becker-Döring cluster equations: Basic properties and asymptotic behavior of solutions, Commun. Math. Phys., 104 (1986), pp. 657-692.

[2] R. BECKER AND W. DÖRING, Kinetische Behandlung der Keimbildung in übersättigten Dämpfen, Annalen der Physik (5. Folge), 24 (1935), pp. 719-752.

[3] D. Brus, V. Ždímal, And J. Smolík, Homogeneous nucleation rate measurements in supersaturated water vapor, J. Chem. Phys., 129 (2008), 174501.

[4] W. Dreyer and F. Duderstadt, On the Becker-Döring theory of nucleation of liquid droplets in solids, J. Stat. Phys., 123:1 (2006).

[5] P. Hartman, Ordinary differential equations, John Wiley \& Sons, Inc. , New York, 1964.

[6] M. Herrmann, M. Naldzhieva, and B. Niethammer, On a thermodynamically consistent modification of the Becker-Döring equations, Physica D, 222 (2006), pp. 116-130.

[7] K. F. Kelton, Crystal nucleation in liquids and glasses, in: H. Ehrenreich and D. Turnbull, Solid state physics, volume 45, Academic Press, Inc., 1991.

[8] P. Laurençot and S. Mischler, From the Becker-Döring to the Lifschitz-Slyozov-Wagner equations, Journal of Stat. Phy., 106:516 (2002).

[9] I. Müller ANd W. H. Müller, Fundamentals of thermodynamics and applications, SpringerVerlag, 2009.

[10] M. NaldZhieva, Die thermodynamisch konsistenten Becker-Döring-Gleichungen, Diploma thesis, Humboldt-Universität zu Berlin. Department of Mathematics, 2006.

[11] O. Penrose, Metastable states for the Becker-Döring cluster equations, Commun. Math. Phys., 124 (1989), pp. 515-541.

[12] S. Sinha, A. Bhabhe, H. Laksmono, J. Wölk, R. Strey, and B. Wyslouzil, Argon nucleation in a cryogenic supersonic nozzle, J. Chem. Phys., 132 (2010), 064304.

[13] V. Ssemaganda, The dynamics of the Becker-Döring model of nucleation, Phd thesis, Ottovon-Guericke University Magdeburg. Faculty of Mathematics, 2011.

[14] J. WöLK AND R. Strey, Homogeneous nucleation of $\mathrm{H}_{2} \mathrm{O}$ and $\mathrm{D}_{2} \mathrm{O}$ in comparison: The Isotope Effect, J. Phys. Chem. B, 105 (2001), pp. 11683-11701. 